\title{
A Note on Optimal Partial Triallel Cross Designs
}

\author{
Anshula Pandey ${ }^{1}$, Addisu Jember ${ }^{2}$ and MK Sharma ${ }^{1 *}$ \\ ${ }^{1}$ Department of Statistics, Sunandan Divatia School of Science NMIMS, India \\ ${ }^{2}$ Addis Ababa University, Addis Ababa, Ethiopia
}

Submission: March 26, 2018; Published: July 09, 2018

*Corresponding author: Anshula Pandey, Department of Statistics, Sunandan Divatia School of Science NMIMS, Mumbai, India; Email: mk_subash@yahoo.co.in

\begin{abstract}
Summary
Two simple alternative methods of construction of optimal partial triallel cross designs (unblocked and blocked) for $\mathrm{p}>3$ lines are proposed by using transversal and parallel transversals of a Latin square of order $p$, where $p$ is a prime or power of a prime. Sharma et al. [1] and Sharma \& Fanta [2] also constructed these designs by using two mutually orthogonal Latin squares but we constructed these designs only taking one Latin square. Therefore our methods are simple in comparison to their methods. These designs are found to be optimal in the sense of Kiefer [3] and Das \& Gupta [4].

Keywords: Partial triallel cross; Transversal; Parallel transversals; Mutually Othogonal; Latin Squares; Mating design; Cross designs; Mating designs; Randomized complete; Homogeneous blocks; Unblocked designs; Optimal block; Three-way hybrids; Half-parents; Triplet; Offspring;
\end{abstract} Directrix; cyclically mod; Parallel transversals

Abbreviations: CRD: Completely Randomized Design; RCB: Randomized Complete Block; PTC: Partial Triallel Crosses; BIB: Balanced Incomplete Block; GPBIBD: Generalized Partially Balanced Incomplete Block Designs

\section{Introduction}

Triallel crosses form an important class of mating designs, which are used for studying the genetic properties of a set of inbred lines in plant breeding experiments. For $\mathrm{p}$ inbred lines, the number of different crosses for a complete triallel experiment is $3^{p} C_{3}=p(p-1)(p-2) / 2$ of the type $(i \times j) k$, $i \neq j \neq k=0,1,2, \ldots, p-1$. Rawlings \& Cockerham [5] were the first to introduce mating designs for triallel crosses. Triallel cross experiments are generally conducted using a completely randomized design (CRD) or a randomized complete block (RCB) design as environmental design involving $3{ }^{p} C_{3}$ crosses. Even with a moderate number of parents, say $p=10$, in a triallel cross experiment; the number of crosses becomes unmanageable to be accommodated in homogeneous blocks. For such situations, Hinkelmann [6] developed partial triallel crosses (PTC) involving only a sample of all possible crosses by establishing a correspondence between PTC and generalized partially balanced incomplete block designs (GPBIBD). Ponnuswamy \& Srinivasan [7] and Subbrayan [8] obtained PTC using a class of balanced incomplete block (BIB) designs. Dharmlingum [9] also constructed PTC using Trojan squares. Actually Trojan squares are MOLS. Other research workers who contributed in this area are Arora \& Aggarwal [10,11], Ceranka et al. [12]. More details on triallel cross experiments can be found in Hinkelmann [13] and Narain [14].
Das \& Gupta [4] constructed block designs for triallel crosses by using nested balanced block design with parameters $v=p, b_{1}, b_{2}, k_{1}, k_{2}=3$. Their method yields designs which are universally optimal in $D(p, b, k)$, the class of connected block designs for triallel crosses in $\mathrm{p}$ lines with b blocks each of size $\mathrm{k}$ such that the total number of experimental units are $<3^{p} C_{3}$. Sharma et al. [1] and Sharma and Fanta [2] also constructed optimal block and unblocked designs for PTC using two mutually orthogonal Latin squares together. We, in this paper, are proposing unblocked and blocked designs for triallel cross experiments by using transversal and parallel transversal of a Latin square. Our methods are different from Sharma et al. [1] and Sharma \& Fanta [2] methods. These designs are found to be optimal in the sense of Kiefer [14] and Das \& Gupta [4].

\section{Definition}

Definition 2.1: The triallel cross (T.C.) has been defined by Rawlings \& Cockerham [5] as a set of all possible three-way hybrids among a group of (inbred) lines. Given three lines $i, j$ and $k$, there are distinct triallel crosses, namely $(i \times j) k,(j \times k) i$ and $(i \times k) j$ involving these three lines. Thus given a set of $p$ lines, the TC will consist of a set of $[p(p-1)(p-2) / 2]$ distinct three way crosses. Each line will occur in $r_{H}=(p-1)(p-2)$ three-way crosses as a half -parents and in $r_{F}=(p-1)(p-2)$ three way crosses as a 
parent. Similarly, each pair of lines will occur in $r_{d}=(p-2)$ threeway crosses both of them as half-parents and $r_{s}=(p-2)$ three way crosses with one of them as half-parent and other as a parent.

Definition 2.2: Hinkelmann [6] proposed the definition of PTC as given below:

Suppose we have $p$ lines which are denoted by $i=1,2, \ldots, p$. A three way cross is then represented by a triplet $(i \times j) k$, where $(i \times j)$ stands for an offspring of the single cross $i \times j$. We shall call $i$ and $j$ half-parents and $k$ full-parent. The crosses $(i \times j) k$, $(j \times i) k, k(i \times j)$, and $k(j \times i)$ are considered to be identical in three way crosses. Then PTC can be defined as follows:

A set of matings is said to be a PTC if it satisfies the following conditions:

Each line occurs exactly $r_{H}$ times as half-parent and $r_{F}$ times as full parent.

Each cross $(i \times j) k$ occurs either once or not at all.

The total number of crosses is $p \quad r_{F}$ and $r_{H}=2 r_{F}$. Let $r_{F}=r$, whence $r_{H}=2 r$.

\section{Construction of partial triallel cross}

Method 1: The first method of construction of PTC mating design is based on transversal (directrix) of Latin square of order $p$, where $p$ is a prime or power of prime. Start with a Latin square of order $\mathrm{p}$ and border the columns and the rows of square with elements of the transversal (directrix) (where transversal or directrix of a Latin square of order $p$ in a set of p cells each row, column and symbol is represented exactly once in the set; see Hedayal \& Seiden [15]. Each cell together with the border elements give a triplet of the type $(i \times j) \times k$, where $i \neq j \neq k=0,1, \ldots \ldots \ldots, p-1$ in the upper and lower triangular and triplet of the type $(i \times j) \times k$ where $i=j=k=0,1 \ldots \ldots \ldots, p-1$ in the diagonal of the mating design. Now deleting the triplet appearing in the diagonal of the mating design we get a mating design for PTC experiment which satisfy the condition of PTC. The method is illustrative below with example.

Example 1: Consider the following Latin square of order 5 transversal bracketed.

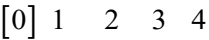
1 [2] 3 (2) 40

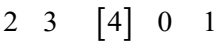

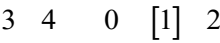
$\begin{array}{lllll}4 & 0 & 1 & 2 & {[3]}\end{array}$

Now we border the columns and rows of the above Latin square by the bracketed elements of the transversal. We get the following arrangement.

Table 1: Now we attach border elements with each cell.

\begin{tabular}{|l|l|l|l|l|l|}
\hline & $\mathbf{0}$ & $\mathbf{2}$ & $\mathbf{4}$ & $\mathbf{1}$ & $\mathbf{3}$ \\
\hline 0 & 0 & 1 & 2 & 3 & 4 \\
\hline
\end{tabular}

\begin{tabular}{|l|l|l|l|l|l|}
\hline 2 & 1 & 2 & 3 & 4 & 0 \\
\hline 4 & 2 & 3 & 4 & 0 & 1 \\
\hline 1 & 3 & 4 & 0 & 1 & 2 \\
\hline 3 & 4 & 0 & 1 & 2 & 3 \\
\hline
\end{tabular}

Now we attach border elements with each cell (Table 1). Then we get the following design

$$
\begin{array}{ccccc}
{[(0 \times 0) 0]} & (0 \times 1) 2 & (0 \times 2) 4 & (0 \times 3) 1 & (0 \times 4) 3 \\
(2 \times 1) 0 & {[(2 \times 2) 2]} & (2 \times 3) 4 & (2 \times 4) 1 & (2 \times 0) 3 \\
(4 \times 2) 0 & (4 \times 3) 2 & {[(4 \times 4) 4]} & (4 \times 0) 1 & (4 \times 1) 3 \\
(1 \times 3) 0 & (1 \times 4) 2 & (1 \times 0) 4 & {[(1 \times 1) 1]} & (1 \times 2) 3 \\
(3 \times 4) 0 & (3 \times 0) 2 & (3 \times 1) 4 & (3 \times 2) 1 & {[(3 \times 3) 3}
\end{array}
$$

Now deleting the bracketed cells, we get following unblocked design $\mathrm{d}_{1}$ for PTC in which

i. Each line occurs $r_{H}(=8)$ lines as half parent and $r_{F}(=4)$ lines as full parent.

ii. Each triple cross $(i \times j) \times k$ either once or not at all in $d_{1}$.

iii. We denote the unblocked design as $d_{1} \varepsilon D(p, n)$ with $p$ lines and $n$ experimental units.

$$
\begin{aligned}
& \text { Design } d_{1} \\
& \begin{array}{lllll}
(2 \times 1) 0 & (0 \times 1) 2 & (0 \times 2) 4 & (0 \times 3) 1 & (0 \times 4) 3
\end{array} \\
& \begin{array}{lllll}
(4 \times 2) 0 & (4 \times 3) 2 & (2 \times 3) 4 & (2 \times 4) 1 & (2 \times 0) 3
\end{array} \\
& \begin{array}{lllll}
(1 \times 3) 0 & (1 \times 4) 2 & (1 \times 0) 4 & (4 \times 0) 1 & (4 \times 1) 3
\end{array} \\
& \begin{array}{lllll}
(3 \times 4) 0 & (3 \times 0) 2 \quad(3 \times 1) 4 & (3 \times 2) 0 \quad(1 \times 2) 3
\end{array}
\end{aligned}
$$

From the above mating design, we can derive block design for PTC. Consider the triplet of any column of the above mating designs as initial blocks and then developing row-wise cyclically mod (5) these blocks, we get block design for PTC. We denote this design $d_{2} \varepsilon D(p, b, k)$ with $p=5, b=4$ and $k=5$.

Consider the first column triplets as 4 initial blocks and then developing cyclically mod (5), we get the following block design for PTC, which satisfy the condition of PTC.

\section{$\operatorname{Design} d_{2}$}

$\begin{array}{llllll}B_{1} & (2 \times 1) 0 & (3 \times 2) 1 & (4 \times 3) 2 & (0 \times 4) 3 & (1 \times 0) 4 \\ B_{2} & (4 \times 2) 1 & (0 \times 3) 1 & (1 \times 4) 2 & (2 \times 0) 3 & (3 \times 1) 4 \\ B_{3} & (1 \times 3) 0 & (2 \times 4) 1 & (3 \times 0) 2 & (4 \times 1) 3 & (0 \times 2) 4 \\ B_{4} & (3 \times 4) 0 & (4 \times 0) 1 & (0 \times 1) 2 & (1 \times 2) 3 & (2 \times 3) 4\end{array}$

Example 2: Now consider the following Latin square of order 5 transversal bracketed.

$\begin{array}{ccccc}{[1]} & 2 & 3 & 4 & 5 \\ 3 & {[4]} & 5 & 1 & 2 \\ 5 & 1 & {[2]} & 3 & 4 \\ 2 & 3 & 4 & {[5]} & 1 \\ 4 & 5 & 1 & 2 & {[3]}\end{array}$


Following the procedure of example 1, we get following unblocked mating design $d_{3} \varepsilon D(p, n)$ for PTC with $p=5$ and $n=20$ experimental units.

\section{Design $d_{3}$}

$\begin{array}{lllll}(4 \times 3) 1 & (1 \times 2) 4 & (1 \times 3) 2 & (1 \times 4) 5 & (1 \times 5) 3 \\ (2 \times 5) 1 & (2 \times 1) 4 & (4 \times 5) 2 & (4 \times 1) 5 & (4 \times 2) 3 \\ (5 \times 2) 1 & (5 \times 3) 4 & (5 \times 4) 2 & (2 \times 3) 5 & (2 \times 4) 3 \\ (3 \times 4) 1 & (3 \times 5) 4 & (3 \times 1) 2 & (3 \times 2) 5 & (5 \times 1) 3\end{array}$

By observing the above design we find that every triple cross occurs two times in the design. Hence this design is different from the design given in example 1. Again by taking triplets of any column as initial blocks and developing each triplet cyclically rowwise $\bmod (5)$ we obtain block design $d_{4}$ with parameters $p=5$, $b=4$ and $k=5$ in which each triple cross is also replicated two times in the design.

$\begin{array}{cccccc}\text { Design } & d_{4} & & & & \\ B_{1} & (4 \times 3) 1 & (5 \times 4) 2 & (1 \times 5) 3 & (2 \times 1) 4 & (3 \times 2) 5 \\ B_{2} & (2 \times 5) 1 & (3 \times 1) 2 & (4 \times 2) 3 & (5 \times 3) 4 & (1 \times 4) 5 \\ B_{3} & (5 \times 2) 1 & (1 \times 3) 2 & (2 \times 4) 3 & (3 \times 5) 4 & (4 \times 1) 5 \\ B_{4} & (3 \times 4) 1 & (4 \times 5) 2 & (5 \times 1) 3 & (1 \times 2) 4 & (2 \times 3) 5\end{array}$

We see that unblocked design $d_{1}$ is different from unblocked design $d_{3}$ and blocked design $d_{2}$ is different from blocked design $d_{4}$. Thus we get two different (unblocked and blocked) designs by using transversal of two Latin squares of order 5 which are mutually orthogonal.

a) Remark 1: It is impossible to construct PTC from Latin square of order 6 because the Latin square of order 6 does not contain distinct elements in the transversal (directrix).

b) Remark 2: we know that when $p$ is a prime or power of a prime then there exist $p-1$ or $p^{n}-1$ mutually orthogonal Latin squares. Out of $p-1$ or $p^{n}-1$ m.o.l.s., we can use only transversals of $p-2$ or $p^{n}-2$ Latin squares for the construction of mating designs for PTC because transversal of only one Latin square does not contain distinct elements.

c) Remark 3: Out of $p-2$ or $p^{n}-2$ Latin squares only one Latin square will give mating design for PTC in which every triple cross occurs two times in unblocked and blocked designs.

Method 2: Two transversals in a Latin square of order $p$ are called parallel if they have no element in common. Suppose we have identified such parallel transversals in a Latin square and border the columns and rows of a Latin square with the elements of parallel transversals for example if the parallel transversal element $a_{i j}$ belong to $i^{\text {th }}$ row and $j^{\text {th }}$ column then this element will border the $i^{\text {th }}$ row and $j^{\text {th }}$ column of the Latin square. Thus we get triallel cross mating design with the difference that triplet of the type $(i \times j) \times k$ where $i=j=k=0,1 \ldots \ldots \ldots, p-1$ occurs on the positions of parallel transversal elements and triplet of the type $(i \times j) \times k$, where $i \neq j \neq k=0,1 \ldots \ldots \ldots, p-1$ occurs below and upper positions of parallel transversals.

Now deleting the triplet appearing at the parallel transversal positions in the triallel cross mating design we get a mating design $d_{5}$ for PTC experiment which satisfy the condition of PTC. The method is illustrative below with example.

Example 3: Consider the following $5 \times 5$ Latin square with two parallel transversals with square bracketed:

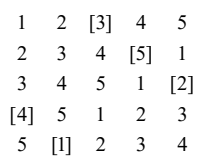

Now we border the columns and rows of the above Latin square by the bracketed elements of the parallel transversals following the rule given in method 2. We get the following arrangement.

Now we attach border elements with each cell. Then we get the following triallel cross mating design

$$
\begin{array}{ccccc}
(3 \times 1) 4 & (3 \times 2) 1 & [3 \times 3) 3] & (3 \times 4) 5 & (3 \times 5) 2 \\
(5 \times 2) 4 & (5 \times 3) 1 & (5 \times 4) 3 & \llbracket 5 \times 5) 5] & (5 \times 1) 2 \\
(2 \times 3) 4 & (2 \times 4) 1 & (2 \times 5) 3 & (2 \times 1) 5 & \llbracket 2 \times 2) 2] \\
[4 \times 4) 4] & (4 \times 5) 1 & (4 \times 1) 3 & (4 \times 2) 5 & (4 \times 3) 2 \\
(1 \times 5) 4 & [1 \times 1) 1] & (1 \times 2) 3 & (1 \times 3) 5 & (1 \times 4) 2
\end{array}
$$

Now deleting the bracketed cells, we get following unblocked design $\mathrm{d}_{5}$ for PTC in which

Each line occurs $r_{H}(=8)$ lines as half parent and $r_{F}(=4)$ lines as full parent.

Each triple cross $(i \times j) \times k$ either once or not at all in $d_{5}$.

\section{Design $d_{5}$}

$\begin{array}{lllll}(3 \times 1) 4 & (3 \times 2) 1 & (5 \times 4) 3 & (3 \times 4) 5 & (3 \times 5) 2\end{array}$

$\begin{array}{lllll}(5 \times 2) 4 & (5 \times 3) 1 & (2 \times 5) 3 & (2 \times 1) 5 & (5 \times 1) 2\end{array}$

$\begin{array}{lllll}(2 \times 3) 4 & (2 \times 4) 1 & (4 \times 1) 3 & (4 \times 2) 5 & (4 \times 3) 2\end{array}$

$\begin{array}{lllll}(1 \times 5) 4 & (4 \times 5) 1 & (1 \times 2) 3 & (1 \times 3) 5 & (1 \times 4) 2\end{array}$

From the above mating design, we can derive blocked mating design for PTC. Consider any column of the above mating designs as initial blocks and then developing these blocks cyclically row wise $\bmod (5)$, we get blocked mating design $d_{6}$ for PTC with parameters $p=5, b=4$ and $k=5$

\section{Design $d_{6}$}

$$
\begin{array}{llllll}
B_{1} & (3 \times 1) 4 & (4 \times 2) 5 & (5 \times 3) 1 & (1 \times 4) 2 & (2 \times 5) 3 \\
B_{2} & (5 \times 2) 4 & (1 \times 3) 5 & (2 \times 4) 1 & (3 \times 5) 2 & (4 \times 1) 3 \\
B_{3} & (2 \times 3) 4 & (3 \times 4) 5 & (4 \times 5) 1 & (5 \times 1) 2 & (1 \times 2) 3 \\
B_{4} & (1 \times 5) 4 & (2 \times 1) 5 & (3 \times 2) 1 & (4 \times 3) 2 & (5 \times 4) 3
\end{array}
$$

Sharma et al. [1] and Sharma \& Fanta [2] constructed these designs by using two M.OL.S. of order $\mathrm{p}$ together, where $\mathrm{p}$ is a prime or prime power but our method needs only one Latin square.

d) Remark 4: When we take parallel transversals out of $p-2$ or $p^{n}-2$ Latin squares only one Latin squares will give mating design for PTC in which every triple cross occurs two times in unblocked and blocked designs.

\section{References}

1. Sharma MK, Asedewoin A, Misganaw B (2011) Optimal partial triallel cross designs. MASA 6: 1-8. 
2. Sharma, M.K. and Fanta, S. (2012). Optimal partial triallel crosses. Jour Indian Society of Ag Statistics 66(2): 1-6.

3. Kiefer J (1975) Construction and optimality of generalized Youden designs, in a survey of Statistical Design and Linear Models. In: Srivastava JN (Eds.), Amesterdam: North Hollond, Netherlands, pp. 3353.

4. Das A, Gupta S (1997) Optimal block designs for triallel cross experiments. Commu Stat-theory and Methods 26(7): 1767-1777.

5. Rawlings J0, Cockerham CC (1962) Triallel analysis. Crop Sci 2: 228231.

6. Hinkelmann K (1965) Partial triallel crosses. Sankhya A 27: 173-196.

7. Ponnuswami, K.N. and Srinivasan,M.R. (1991). Construction of partial triallel crosses (PTC) using a class of balanced incomplete block designs (BIBD). Commu Stat-Theory and Methods A20: 3315-3323.

8. Subbarayan A (1992) On the applications of pure cyclic triple system for plant breeding experiments. Jour of Applied Stat 19(4): 489-500.
9. Dharmalingam M (2002) Construction of Partial Triallel Crosses based on Trojan square design. Jour of Applied Stat 29(5): 695-702.

10. Arora BS, Aggarwal KR (1984) Confounded triallel experiments and their applications. Sankhya B 46(1): 54-63.

11. Arora BS, Aggarwal KR (1989) Triallel experiments with reciprocal effects. Jour Ind Soc Agril Stat 41: 91-103.

12. Ceranka B, Chudzik H, Dobek A, Kielczewska H (1990) Estimation of parameters for triallel crosses compared in block designs. Statist Appl 2: 27-35.

13. Hinkelmann K (1975) A Survey of Statistical Design and Linear Models. Amsterdam, North Hollond, Netherlands, Pp. 243-269.

14. Narain P (1990) Statistical Genetics. Wiley Eastern Limited, New Delhi, India.

15. Hedayat AS, Seiden E (1974) On the theory and application of sum composition of latin squares and orthogonal latin squares. Pacific J Math 54: 85-112.

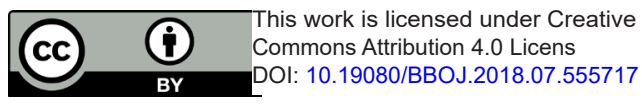

\section{Your next submission with Juniper Publishers will reach you the below assets}

- Quality Editorial service

- Swift Peer Review

- Reprints availability

- E-prints Service

- Manuscript Podcast for convenient understanding

- Global attainment for your research

- Manuscript accessibility in different formats

( Pdf, E-pub, Full Text, Audio)

- Unceasing customer service

Track the below URL for one-step submission https://juniperpublishers.com/online-submission.php 